\title{
Centro Pop e Intersetorialidade: o problema da articulação com a rede de saúde mental
}

\author{
Centro Pop and Intersectorality: \\ the problem of the articulation with the mental health network
}

Tacianna Bandim Pedrosa (https://orcid.org/0000-0001-6579-9800) ${ }^{1}$

Pablo Cáceres-Serrano (https://orcid.org/0000-0002-1691-9199) ${ }^{1}$

${ }^{1}$ Escuela de Psicología Pontificia Universidad Católica de Valparaíso", Campus Sausalito. Avenida El Bosque 1290 Viña del Mar.

Valparaíso Chile.

taciannabp@gmail.com

\begin{abstract}
Mental health care for the homeless population (HP) is an important topic but supported by several studies as underexplored. Given the complexity of this demand, there are several services in the SUS and SUAS that require an intersectoral work articulated in a network; however, studies point out several flaws in their performance. In this secondary statistical study, we propose a discussion of this articulation based on the results of the SUAS 2019 CENSUS. Participants: 228 Centro Pops, from 25 Brazilian states. Variables from the block of questions related to the articulation of the Centro Pop with CAPS and hospitals were chosen for analysis and the Multiple Correspondence Analysis (MCA) was used to establish associations between variables and their categories. The results show a moderately satisfactory articulation with the CAPS and unsatisfactory in relation to the hospitals. It is concluded that the articulation of the Centro Pop with the CAPS is relevant mainly in large municipalities, and incipient with hospitals, regardless of the population size. The flow protocol is highlighted as an important tool for effective intersectorality in health care for the HP and the right to the city, and longitudinal studies on the subject are suggested, to aid in decision-making instances.

Key words Homeless, Intersectorality, Mental health, Psychosocial care, Networks
\end{abstract}

Resumo O cuidado em saúde mental para a população em situação de rua (PSR) é um tema importante, mas sustentado por diversos estudos como subexplorado. Diante da complexidade desta demanda, temos diversos serviços no Sistema Único de Saúde (SUS) e no Sistema Único de Assistência Social (SUAS) que requerem trabalho intersetorial articulado em rede, não obstante, estudos apontam variadas falhas em sua execução. Neste estudo estatístico secundário, propomos a discussão desta articulação baseada nos resultados do Censo SUAS 2019. Participantes: 228 Centros Pop, de 25 estados brasileiros. Escolhidas para análise as variáveis do bloco de questões referentes à articulação do Centro Pop com CAPS e hospitais e utilizada a Análise de Correspondência Múltipla (ACM) para estabelecer associações entre variáveis e suas categorias. Resultados apontam articulação moderadamente satisfatória com os CAPS e insatisfatória em relação aos hospitais. Conclui-se que a articulação do Centro Pop com o CAPS é relevante principalmente nos municipios de grande porte e incipiente com os hospitais, independentemente do porte populacional. Destaca-se o protocolo de fluxo como importante ferramenta para a efetiva intersetorialidade na atenção em saúde à PSR e no direito à cidade e sugere-se estudos longitudinais sobre o tema, para auxílio na tomada de decisão. Palavras-chave População em situação de rua, Intersetorialidade, Saúde mental, Atenção psicossocial, Redes 


\section{Introdução}

A caracterização da população em situação de rua (PSR) é marcada dentre outros fatores, por grande vulnerabilidade social e complicações de saúde, principalmente, o uso abusivo de psicoativos e sofrimento psíquico intenso ${ }^{1-3}$. A Reforma Psiquiátrica no contexto brasileiro gerou uma grande mudança na vida de pessoas com intenso sofrimento psíquico, especialmente aquelas que se encontravam institucionalizadas por longa data, mudando o paradigma até então estabelecido da lógica manicomial, para a lógica do cuidado em liberdade sendo a expressão máxima dessa reorientação, a promulgação da lei 10.216/014.

Esta mudança epistemológica é responsável por fomentar a base na qual a política pública de saúde mental é fundada no Brasil e, ao mesmo tempo, reflete em outras políticas públicas que resguardam aproximações com a Rede de Atenção Psicossocial (RAPS) - Portaria GM/MS no 3.088 de 23/12 de 2011 - e possuem necessidade de retroalimentação mútua e trabalho intersetorial. É o caso, por exemplo, da atenção à saúde da PSR ${ }^{3}$. Desta forma, há um importante desafio na atenção a esta população, para a política pública de saúde mental brasileira, sobretudo para a RAPS e especialmente para os Centros de Atenção Psicossocial (CAPS), considerando os princípios de universalidade, equidade e integralidade do Sistema Único de Saúde (SUS), que prevê o acesso à saúde para todos os brasileiros sem distinção $0^{5}$.

No campo social, a luta por direitos à PSR ganha força de lei, quando a Lei Orgânica da Assistência Social (LOAS) é alterada no Brasil e passa a incluir sob sua égide, as pessoas em situação de rua em 2005. Nesse contexto, no marco da criação da Política Nacional de Pessoas em Situação de Rua ${ }^{6}$ são criados os Centros Pop, serviços que atuam como porta de entrada para a PSR na rede de atenção de média complexidade do Sistema Único de Assistência Social (SUAS) e possíveis facilitadores das ações intersetoriais para o campo ${ }^{7,8}$.

O Centro Pop é um Centro de Referência Especializado para a PSR, que atende famílias e indivíduos ofertando desde escuta e acolhimento inicial, até a atenção a necessidades básicas imediatas como: banho, alimentação, espaço para lavar roupas e encaminhamentos para a confecção de documentos de identificação. As principais dimensões do trabalho no Centro Pop são: acolhimento, acompanhamento especializado e articulação em rede, ou seja, trabalho intersetorial ${ }^{7}$. É importante mencionar que a demanda intersetorial por cuidados a esta população, é discutida há algumas décadas, como sugerido por pesquisa publicada em $1998^{9}$ :

Mesmo reconhecendo que o efetivo estabelecimento de uma politica articulada entre o poder público e a sociedade civil tem sido a tônica de vários discursos, como a maneira mais eficaz de intervenção nesta situação social, permanece como grande desafio para os diferentes agentes sociais a construção de uma rede de serviços, constituída de instituições públicas e privadas, consubstanciando parcerias efetivas com o Estado, como a resposta assistencial mais adequada à esse grupo populacional (p. 61).

No caso do cuidado em saúde à PSR, a literatura nacional continua marcando a necessidade de desenvolvimento e fortalecimento de uma rede intersetorial para proporcionar melhor atenção ${ }^{10}$. No entanto, o conceito de intersetorialidade aparece como um problema em sua própria terminologia, devido à sua polissemia ${ }^{11,12}$. Desta forma, buscando minimizar este impasse para o fim específico desta discussão, utilizaremos o conceito de intersetorialidade presente em Junqueira ${ }^{13}$ : "É uma nova lógica para a gestão da cidade, buscando superar a fragmentação das políticas, ao considerar o cidadão na sua totalidade. Isso passa pelas relações homem/natureza e homem/homem que determinam a construção social da cidade" (p. 42). Em suma é, "garantir o acesso igualitário aos desiguais"13.

Com a tendência de concentração da PSR nos grandes centros urbanos ${ }^{7,14}$ a atenção a esta população torna-se ainda mais complexa, dada as dificuldades em mobilizar-se nas metrópoles e grandes cidades e os deslocamentos inerentes à $\mathrm{PSR}^{8}$, somado a isso, pessoas em situação de rua geralmente buscam os serviços de saúde quando sua condição física atinge níveis críticos, resultando em demanda por atenção emergencial $^{8,14,15}$. Relacionadas estas problemáticas, nos perguntamos como os serviços de atenção à PSR se articulam para atender as necessidades de saúde desta população, com ênfase na saúde mental e na atenção emergencial pensando no acesso à saúde para esse seguimento populacional, como uma premissa do direito à cidade ${ }^{16}$.

Apesar do tema da atenção à saúde da PSR no contexto da RAPS, apresentar-se socialmente relevante, poucos estudos são encontrados, sobressaindo-se os estudos qualitativos, exploratórios e de pequeno alcance, uma vez que o foco está em poucas equipes e serviços ${ }^{3}$. Diante do exposto, justificamos a relevância do presente estudo com a proposta de realizar uma análise quantitativa da articulação intersetorial entre Centro 
Pop e serviços de atenção em saúde mental (incluindo hospital geral), utilizando os dados do Censo SUAS 2019, na tentativa de tornar visíveis algumas lacunas ainda existentes em termos de intersetorialidade entre atenção em saúde mental e sua intersecção com o Sistema Único de Assistência Social (SUAS).

\section{Método}

\section{Participantes}

228 Serviços de atendimento à população de rua do Sistema Único de Assistência Social do Brasil (Centros Pop). A pesquisa contou com a participação dos serviços de 25 unidades da federação.

\section{Desenho}

Não-experimental, transseccional, retrospectiva.

\section{Instrumentos}

Este estudo é uma análise secundária que utiliza o banco de dados do Censo SUAS 2019 ${ }^{17}$, disponível em Excel na página do Ministério da Cidadania, antigo "Ministério de Desenvolvimento Social", específico do Centro Pop, obtido a partir da aplicação do questionário CENSO SUAS $^{18}$, que é uma pesquisa censitária, realizada em todos os estados e distrito federal do país, excluindo apenas os estados de Roraima e Tocantins que em 2019, não possuíam um Centro Pop. O CENSO SUAS é realizado anualmente com o Centro Pop, desde 2011.

Utilizado como questionário administrativo para avaliação dos serviços, composto por sete blocos de questões: 1) Identificação; 2) Estrutura Física; 3) Atendimento especializado para pessoas em situação de rua; 4) Atendimento especializado em abordagem social; 5) Benefícios, cadastro único e participação do usuário; 6) Articulação; 7) Recursos Humanos (Ministério da Cidadania, Secretaria Nacional de Assistência Social, 2019). Não foram apontados erros na condução do questionário geral, validando a partir desse fato, o instrumento.

Posteriormente, foi utilizada a Análise de Correspondência Múltipla (ACM) para reduzir as informações e estabelecer associações entre variáveis e suas categorias. A ACM é uma técnica de interdependência que permite estabelecer associações entre as variáveis qualitativas incluídas no estudo, bem como associações que possam surgir entre categorias da mesma variável ou diferentes variáveis ${ }^{19}$. Como análise dos principais componentes, é também uma técnica de redução da informação ${ }^{20}$.

\section{Procedimento}

O CENSO SUAS ${ }^{17}$ foi realizado pela Coordenação Geral de Planejamento e Vigilância Socioassistencial do Departamento de Gestão do SUAS. Realizamos a busca pela base de dados que se encontra disponível no site do Ministério da Cidadania, como informação pública. Após a obtenção de todos os arquivos, referentes ao Centro Pop, selecionamos as variáveis relacionadas aos serviços CAPS e serviços de urgência e emergência (hospitais) do bloco 6 "Articulação", tomando em consideração também o porte populacional dos municípios (Grande, Médio e Metrópole) e obtivemos um total de 24 variáveis.

Em primeiro lugar, foi realizada uma análise descritiva de todas as 24 variáveis selecionadas no questionário aplicado, mas decidiu-se eliminar todas aquelas em que uma das categorias de resposta foi respondida por um percentual igual ou superior a 90\%. Essas questões apresentaram pouca variabilidade e não contribuíram para a análise. Consequentemente, apenas 15 variáveis foram incluídas na análise subsequente. Como as variáveis são dicotômicas, apenas frequências e percentuais são relatados.

A Análise de Correspondência Múltipla (ACM) é uma técnica de interdependência multivariada, por vezes entendida como uma generalização de uma representação gráfica conhecida como diagrama de dispersão ${ }^{19}$. Permite descrever a associação entre variáveis categóricas, como questões nominais de múltipla escolha ou dicotômicas, como as respondidas com um "Não", denominado mapa perceptivo.

Os testes de significância estatística não são usados na ACM, o método envolve a construção de uma tabela de contingência composta por "i" linhas e “j” colunas, que dá origem a uma tabela I x J. A partir da tabela, que contém as frequências conjuntas das variáveis e suas categorias, ela é representada no mapa perceptual, as distâncias entre as variáveis e as modalidades das variáveis consideradas na análise, assumindo que uma distância menor implica uma associação entre categorias e uma distância maior, o contrário ${ }^{21}$.

O espaço bidimensional ou espaço fatorial é construído por eixos ou planos de projeção, tomados dois a dois, de um conjunto de fatores que 
foram obtidos na solução preliminar da análise. A escolha dos eixos sobre os quais as variáveis e / ou suas categorias são projetadas é baseada em critérios objetivos e subjetivos. Esses eixos são componentes extraídos das informações disponíveis e reduzem a dimensionalidade como na análise de componentes principais, permitindo explicar a inércia presente nos dados representados no espaço bidimensional formado por tais eixos $^{19}$. Normalmente, os eixos que explicam a maior porcentagem de variação são selecionados.

Uma vez que os eixos ou dimensões fatoriais tenham sido escolhidos e as observações e as variáveis e suas modalidades ou categorias tenham sido projetadas sobre eles, será necessário estudar o quanto cada modalidade contribui para a variabilidade do eixo e quão bem estão representadas as observações e/ou modalidades pelos referidos eixos $^{21}$. A qualidade da representação geralmente é avaliada com base no cálculo dos cossenos quadrados entre categoria e eixo, assim, maior valor, melhor representação $0^{22}$.

Se a representação for aceitável, a interpretação dos resultados faz-se por meio de aproximação gráfica, observando o mapa perceptivo construído, sem prejuízo de complementar tal interpretação com os resultados quantitativos que acompanham a análise. O presente estudo utiliza a ACM baseado no trabalho de Benzecri ${ }^{19}$ e seus correspondentes procedimentos analíticos, implementados no pacote de análise FactoMineR ${ }^{22}$.

Operacionalmente, o primeiro passo consiste em a) selecionar as variáveis que podem ser associadas e consequentemente podem contribuir mais fortemente para gerar os eixos com os quais construir o espaço fatorial onde posicionar as variáveis e suas modalidades; A segunda etapa consiste em b) aplicar a técnica, considerando a seleção dos eixos mais adequados sobre os quais projetar as categorias para obter sua posição no mapa perceptivo. Normalmente, esse critério nada mais é do que manter os eixos que explicam a maior proporção de variância nos dados. Normalmente são os dois primeiros eixos extraídos ${ }^{21}$.

\section{Resultados}

\section{Análise descritiva}

Em primeiro lugar, são fornecidas as variáveis incluídas na análise, sua frequência e percentual.

A Tabela 1 fornece as frequências de escolha de resposta para cada uma das perguntas do ques- tionário. A coluna rotulada "Chave" é a expressão resumida da variável na análise do mapa (incluir a questão completa interfere na qualidade da representação gráfica). Observa-se que apenas aquelas questões com maior variância foram deixadas, eliminando aquelas em que uma categoria acumula $90 \%$ ou mais das preferências, essas variáveis pouco contribuem para a discriminação entre as entidades. Note que as perguntas sobre o hospital são indicadas pela letra $\mathrm{H}$ no final.

\section{Análise de correspondência múltipla}

Com as variáveis previamente organizadas, foi realizada uma ACM. As duas primeiras dimensões foram preservadas, explicando 50,3\% da variância total. Nem todas as variáveis preservadas também contribuíram para a construção de dimensões, por isso, uma primeira análise consistiu em estudar a alíquota de contribuição dessas variáveis, bem como sua qualidade de representação. Os resultados podem ser vistos na Tabela 2.

A Tabela 2 fornece os resultados da contribuição das categorias de cada variável para as dimensões. Deve-se notar que categorias menos frequentes contribuem mais porque colaboram mais para a inércia (variação) dos eixos, em termos percentuais. Isso explica uma maior contribuição das categorias "Não" que são respostas menos frequentes.

Por sua vez, os cossenos quadrados expressam a qualidade da representação das variáveis nas dimensões extraídas para gerar a solução. Essa qualidade de representação é avaliada de 0 a 1 e são esperados valores próximos à unidade.

Na Tabela 2 é possível observar que a melhor qualidade de representação das variáveis é dada com a dimensão 1 , com exceção das variáveis Reuniões e Atividades Periódicas como um todo, cuja projeção é melhor na dimensão 2. A qualidade de representação deve ser considerada ao interpretar os resultados, já que representações baixas podem levar a soluções equívocas.

Tendo avaliado a contribuição das variáveis e sua qualidade de representação, passamos a estudar os mapas perceptuais que fornecem uma aproximação gráfica das associações entre as variáveis e suas categorias.

O Gráfico 1 possui os dois primeiros eixos ou dimensões em que as variáveis de estudo são observadas. A proximidade das variáveis aos eixos indica sua maior ou menor correlação (o valor do coeficiente é mostrado nos eixos). Pode-se observar que a dimensão 1 tem maiores correlações 
Tabela 1. Descritivos das perguntas incluídas na análise.

\begin{tabular}{llrrrc}
\hline \multirow{2}{*}{ Perguntas } & \multicolumn{1}{c}{ Chave } & \multicolumn{2}{c}{ Não } & \multicolumn{2}{c}{ Sim } \\
\cline { 3 - 5 } & & Frequência & Porcentagem & Frequência & Porcentagem \\
\hline Acompanha as derivações & Acomp_deriv & 45 & $19.7 \%$ & 183 & $80.3 \%$ \\
Reuniões periódicas & Reun_period & 118 & $51.8 \%$ & 110 & $48.2 \%$ \\
Realiza estudos de caso em conjunto & Estudos_caso & 83 & $36.4 \%$ & 145 & $63.6 \%$ \\
Atividades em conjunto & Ativ_conjunto & 108 & $47.4 \%$ & 120 & $52.6 \%$ \\
Possui fluxo & Possui_fluxo & 120 & $52.6 \%$ & 108 & $47.4 \%$ \\
Possui dados de localização (hospital) & Dados_loc & 53 & $23.2 \%$ & 175 & $76.8 \%$ \\
Recebe usuários (hospital) & Recebe_usuariosH & 81 & $35.5 \%$ & 147 & $64.5 \%$ \\
Deriva usuários (hospital) & Deriva_UsuariosH & 77 & $33.8 \%$ & 151 & $66.2 \%$ \\
Acompanha as derivações (hospital) & Acomp_derivH & 113 & $49.6 \%$ & 115 & $50.4 \%$ \\
Reuniões periódicas (hospital) & Reun_periodH & 198 & $86.8 \%$ & 30 & $13.2 \%$ \\
Troca informações (hospital) & Troca_infoH & 72 & $31.6 \%$ & 156 & $68.4 \%$ \\
Estudos de caso em conjunto (hospital) & Estudos_casoH & 167 & $73.2 \%$ & 61 & $26.8 \%$ \\
Atividades em conjunto (hospital) & Ativ_conjuntoH & 186 & $81.6 \%$ & 42 & $18.4 \%$ \\
Possui fluxo hosp & Possui_fluxoH & 170 & $74.6 \%$ & 58 & $25.4 \%$ \\
\hline
\end{tabular}

Fonte: Autores.

Tabela 2. Contribuição para a construção dos eixos e qualidade da representação.

\begin{tabular}{|c|c|c|c|c|c|}
\hline \multirow{2}{*}{ Questão } & \multirow{2}{*}{ Categoria } & \multicolumn{2}{|c|}{ Contribuição } & \multicolumn{2}{|c|}{ Cossenos quadrados } \\
\hline & & Dimensão 1 & Dimensão 2 & Dimensão 1 & Dimensão 2 \\
\hline \multirow[t]{2}{*}{ Acompanha as derivações } & Não & 4.92 & 0.31 & 0.31 & 0.01 \\
\hline & Sim & 1.21 & 0.08 & 0.31 & 0.01 \\
\hline \multirow[t]{2}{*}{ Reuniões periódicas } & Não & 2.39 & 6.24 & 0.25 & 0.27 \\
\hline & Sim & 2.56 & 6.69 & 0.25 & 0.27 \\
\hline \multirow[t]{2}{*}{ Realiza estudos de caso em conjunto } & Não & 3.87 & 5.66 & 0.30 & 0.18 \\
\hline & Sim & 2.21 & 3.24 & 0.30 & 0.18 \\
\hline \multirow[t]{2}{*}{ Atividades em conjunto } & Não & 2.58 & 7.62 & 0.24 & 0.30 \\
\hline & Sim & 2.32 & 6.86 & 0.24 & 0.30 \\
\hline \multirow[t]{2}{*}{ Possui fluxo } & Não & 2.23 & 5.60 & 0.24 & 0.24 \\
\hline & Sim & 2.48 & 6.23 & 0.24 & 0.24 \\
\hline \multirow[t]{2}{*}{ Possui dados de localização } & Não & 6.37 & 7.73 & 0.41 & 0.21 \\
\hline & Sim & 1.93 & 2.34 & 0.41 & 0.21 \\
\hline \multirow[t]{2}{*}{ Recebe usuários (hosp) } & Não & 5.76 & 5.70 & 0.45 & 0.18 \\
\hline & Sim & 3.17 & 3.14 & 0.45 & 0.18 \\
\hline \multirow[t]{2}{*}{ Deriva usuários (hosp) } & Não & 6.56 & 7.13 & 0.49 & 0.22 \\
\hline & Sim & 3.34 & 3.64 & 0.49 & 0.22 \\
\hline \multirow[t]{2}{*}{ Acompanha as derivações (hosp) } & Não & 4.93 & 3.60 & 0.49 & 0.15 \\
\hline & Sim & 4.84 & 3.54 & 0.49 & 0.15 \\
\hline \multirow[t]{2}{*}{ Reuniões periódicas (hosp) } & Não & 0.84 & 0.40 & 0.32 & 0.06 \\
\hline & Sim & 5.51 & 2.67 & 0.32 & 0.06 \\
\hline \multirow[t]{2}{*}{ Troca informações (hosp) } & Não & 6.90 & 5.33 & 0.50 & 0.16 \\
\hline & Sim & 3.18 & 2.46 & 0.50 & 0.16 \\
\hline \multirow[t]{2}{*}{ Estudos de caso em conjunto (hosp) } & Não & 2.12 & 0.17 & 0.40 & 0.01 \\
\hline & Sim & 5.79 & 0.46 & 0.40 & 0.01 \\
\hline \multirow[t]{2}{*}{ Atividades em conjunto (hosp) } & Não & 1.13 & 0.30 & 0.31 & 0.03 \\
\hline & $\operatorname{Sim}$ & 5.02 & 1.32 & 0.31 & 0.03 \\
\hline \multirow[t]{2}{*}{ Possui fluxo (hosp) } & Não & 1.48 & 0.38 & 0.29 & 0.03 \\
\hline & Sim & 4.35 & 1.12 & 0.29 & 0.03 \\
\hline
\end{tabular}

Fonte: Autores. 
com "Acompanha as derivações", "Possui fluxo" $(\mathrm{H})$, "estudos de caso conjuntos" $(\mathrm{H})$ e "atividades conjuntas" $(\mathrm{H})$. As variáveis "atividades conjuntas", "possui fluxo" e "reuniões periódicas" têm uma relação maior com o eixo 2 , o que é consistente com sua qualidade de representação, maior com a dimensão 2. Note que este resultado também indica que aquelas variáveis que estão relacionadas a um mesmo eixo, também se correlacionam entre si. Consequentemente, existe uma associação entre os grupos de variáveis que mencionamos.

O Gráfico 2 mostra as categorias das variáveis no mapa gráfico. Uma escala gradual de cor foi adicionada para indicar a qualidade de representação das categorias $(\cos 2)$.

Um dos aspectos evidentes no mapa é que existem conjuntos de categorias agrupadas por quadrante e que, como as categorias são dicotômicas, com respostas de Sim e Não, os eixos opõem algumas respostas a outras. Assim, por exemplo, no quadrante inferior esquerdo a categoria Troca_infoH_Sim é observada e no quadrante superior direito, Troca_infoH_Não é encontrado, o mesmo acontece com Acomp_derivH_Sim e Acomp_derivH_Nao. Os eixos têm a capacidade de discriminar entre respostas positivas e negativas. Algo semelhante acontece entre os quadrantes inferior direito e superior esquerdo.
No entanto, o aspecto mais importante é que a distância entre as categorias representa uma medida da semelhança ou dissimilaridade entre elas. Assim, por exemplo, no quadrante superior esquerdo observa-se que uma resposta afirmativa para "Possui fluxo", "Reuniões" e "Atividades Periódicas" como um todo, indica uma associação entre eles ou, que uma resposta afirmativa em um deles implica em uma resposta afirmativa nas demais. Não obstante, no lado oposto do eixo (quadrante inferior direito) ocorre o contrário: quem responde "Não" a "possui fluxo", não realiza Reuniões ou Atividades periódicas em conjunto.

Ao observar o quadrante inferior esquerdo em direção ao canto superior direito, a mesma organização pode ser observada: Centros POP que respondem Sim a: Deriva usuários (hosp), também respondem Sim para Recebe usuários (hosp), Troca informações (hosp), Acompanha as derivações (hosp) e Possui dados de localização. No quadrante oposto, superior direito, ocorre o contrário, a proximidade dessas categorias indica sua associação.

Por fim, ao Gráfico 2, adicionaremos o tipo de porte populacional a que pertencem os Centros de POP e tentaremos determinar sua associação com as categorias analisadas anteriormente (Gráfico 3).

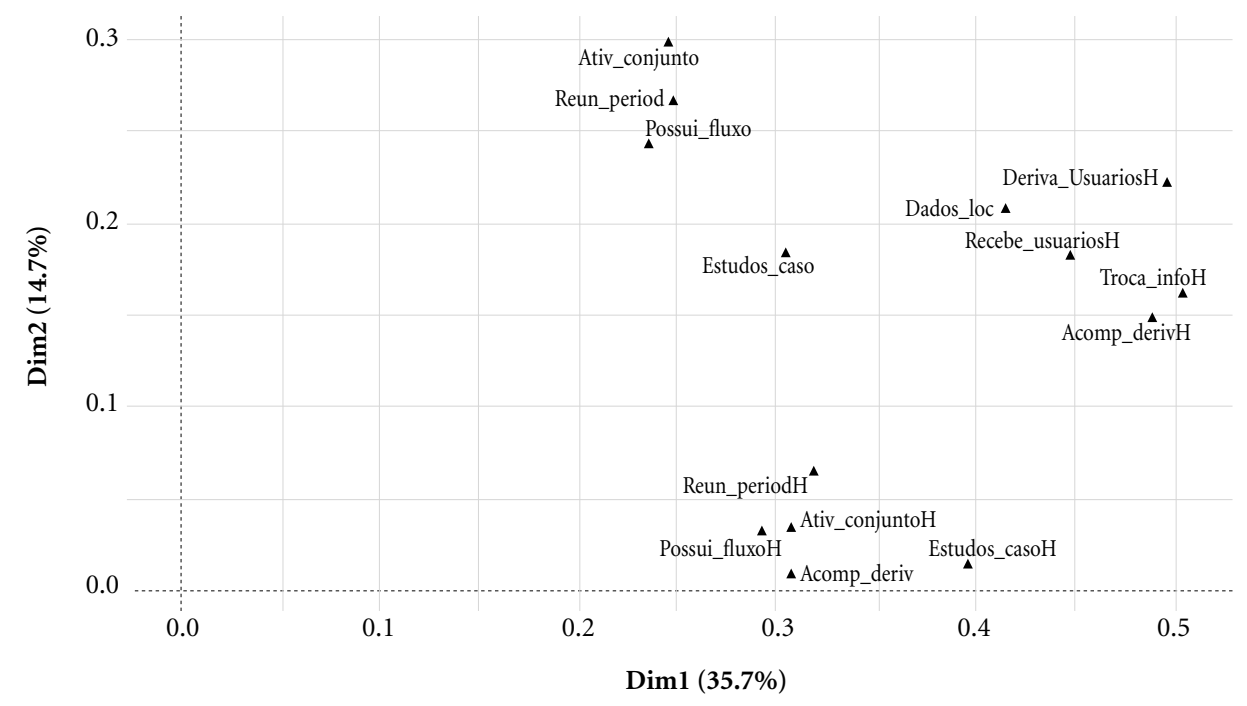

Gráfico 1. Correlação das variáveis com as dimensões. 


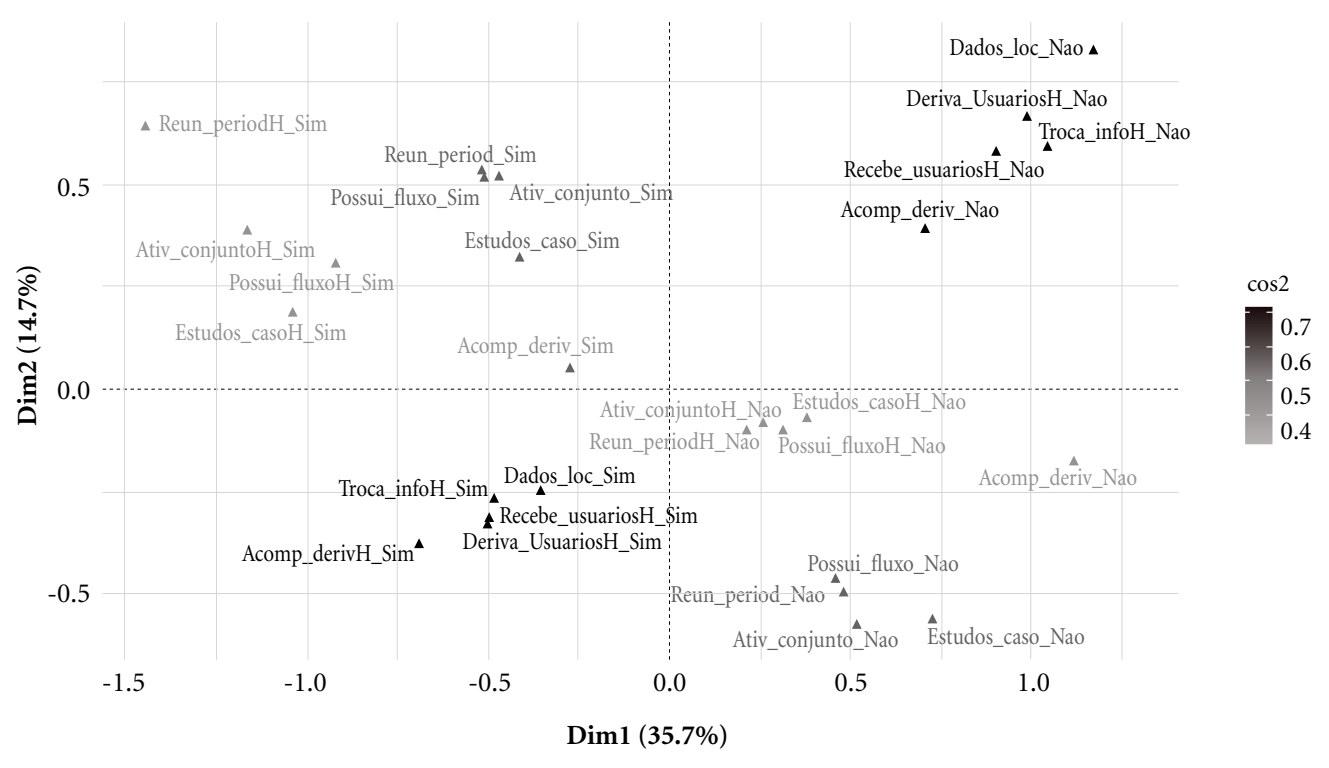

Gráfico 2. Categorias de variáveis no espaço dimensional ACM.

Fonte: Autores.

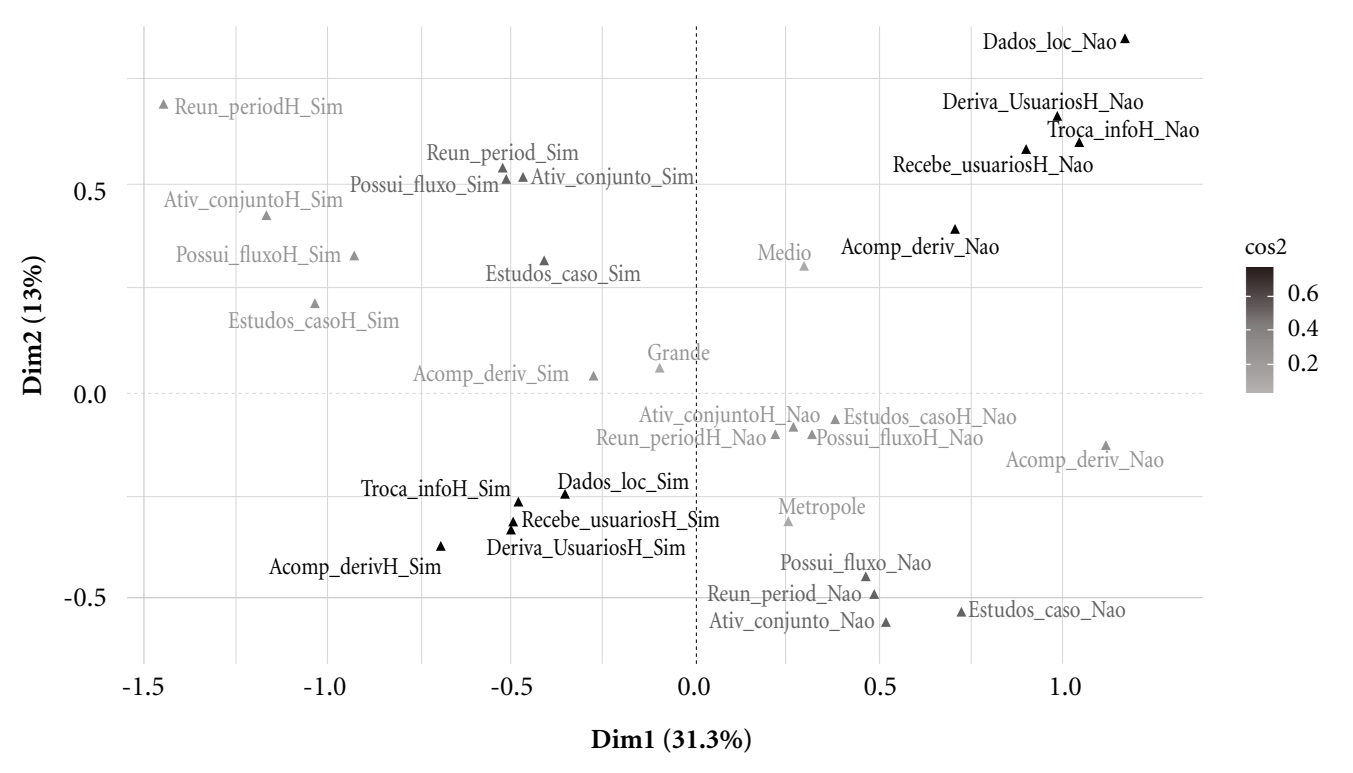

Gráfico 3. Categorias de população e tamanhos no espaço dimensional MCA.

Fonte: Autores. 
Embora infelizmente a qualidade de representação das populações seja baixa, e, portanto, a interpretação feita deste mapa deva ser tomada com cautela, o que se mostra é uma associação entre responder negativamente e em conjunto às questões: "Possui fluxo", "Reuniões periódicas", "Atividades conjuntas" e "Realizar estudos de caso conjuntos" e o tipo de população Metrópole.

Algo semelhante ocorre com o tipo populacional Médio, que está associado a uma resposta negativa a questões como "Deriva usuários (hosp)", "Acompanha as derivações (hosp)", "Recebe usuários (hosp)", "Troca informações (hosp)" " "Possui dados de localização".

Apenas o tipo de grande população está mais associado a uma resposta positiva, como pode ser visto no mapa, porque está no mesmo quadrante superior esquerdo que as respostas positivas.

É importante notar que a associação das categorias com os tipos de populações deve ser interpretada como um todo, por exemplo, no tipo de população Média, percebe-se que uma resposta negativa é dada ao conjunto completo de variáveis próximas, não apenas para alguns.

Consequentemente, nas metrópoles há muitas ações que não são realizadas e o mesmo acontece com os Centros POP das áreas de médio porte, apenas nas áreas de grande porte se cumprem muitas das ações que os Centros de POP devem cumprir. No entanto, deve-se destacar que nas áreas definidas como Grandes, a maior não conformidade está relacionada ao vínculo com o hospital. Algo semelhante acontece com Metrópole, mas em menor grau.

\section{Discussão}

\section{Debilidades e fortalezas nas ações intersetoriais entre Centro Pop e RAPS}

De acordo com os resultados, o Centro Pop aparece com uma resposta positiva expressiva em relação à articulação com os serviços de saúde mental em grande parte das variáveis, destacando que na maior parte dos municípios, há serviços de saúde mental de referência no território onde estão localizados, o que pode refletir a expansão da RAPS nos últimos anos.

Porém, apesar da grande cobertura dos CAPS e da expressiva articulação dos Centros Pop com estes, o fato de haver uma fragilidade importante na articulação com outros serviços como urgência e emergência (hospitais) confirma que:

Apesar dos investimentos positivos e das articulações com as diretrizes da RAPS, são encon- trados obstáculos nesse processo. Algumas equipes relatam dificuldades na articulação do trabalho da rede para responder a emergências e realizar ações complexas, sugerindo integração insuficiente dos pontos da rede assistencial e falta de corresponsabilidade ${ }^{3}$.

Tal fato demonstra que outras políticas públicas têm dificuldade em priorizar a atenção à população de rua, o que também é evidente em outras práticas de saúde: "A crítica dos trabalhadores ao cuidado prestado pelas equipes de saúde do SUS baseia-se na falta de preparo dos profissionais para lidar com as pessoas de rua, no estigma na prestação de cuidados e na discriminação demonstrada pelos profissionais de saúde"23.

No âmbito do interstício entre as políticas públicas responsáveis pela atenção à PSR, a literatura nacional também aponta algumas questões que influenciam na eficácia ou ineficiência da intersetorialidade como a existência de lacunas ${ }^{9}$ na comunicação intersetorial e discordâncias entre os papéis da assistência social e da saúde ${ }^{3,10,22-24}$. O papel da articulação intersetorial para a PSR, por exemplo, é cumprido na maioria das vezes, pelas equipes de assistência social ${ }^{10}$.

Entretanto, segundo Sicari e Zanella ${ }^{25}$ : "o sentimento de crença e confiança nos serviços prestados pelo Centro POP foi identificado nos sem-teto, mas ressalta que esse serviço precisa desenvolver mais ações que atendam às pessoas em sua singularidade, garantindo-lhes direitos básicos e acesso à rede intersetorial" (p. 670). Podemos inferir com este estudo que de fato o Centro Pop possui um papel relevante na articulação com a RAPS, devido às altas respostas positivas principalmente em relação aos CAPS, porém as respostas negativas relacionadas à variável "possui fluxo" entregam pistas sobre um possível problema já relatado por profissionais da área: como restrições aos serviços e ausência de fluxos ${ }^{10}$.

\section{Fluxos como organizadores da rede?}

$\mathrm{O}$ estudo sugere que em alguns casos, a resposta negativa para "Possui fluxo" (H), (principalmente no caso da articulação com os hospitais), está associada com respostas negativas a outras variáveis como "Realiza Reuniões" ou "Atividades periódicas em conjunto". A ausência de fluxos pode estar relacionada a dificuldades de articulação intersetorial e iniquidades de saúde, que também revelam um modelo de sociedade que rejeita os loucos, errantes ou vagabundos de rua. Ressalta-se que nem sempre ter protocolos, ou fluxos instituídos, garante melhor qualidade 
no atendimento, mas não é muito problematizada a ausência do protocolo de fluxo nas políticas públicas para PSR, o que pode ser interpretado como uma naturalização da negação do direito à cidade para este seguimento populacional. Porque esta demanda não necessitaria de fluxos ou protocolos de atenção e cuidado instituídos?

Nos descritores em ciências da saúde da Biblioteca Virtual em Saúde, encontramos a expressão "fluxo de trabalho", que descreve o que estamos considerando como "protocolo de fluxo": "descrição do padrão de funções ou procedimentos recorrentes que muitas vezes são encontrados em processos organizacionais, como notificação, decisão e ação. Sinônimos: Flowchart; Modelagem de processos; fluxo de trabalho, ou, Fluxo de Trabalho".

Conforme esta descrição, entendemos ter um papel importante a instituição de fluxos entre os serviços, como garantia de acesso e demonstração de corresponsabilidade, uma vez que há indícios de falta de fluxos para atenção à PSR ${ }^{10,25,26}$. Por outro lado, talvez se necessite problematizar a tendência brasileira em articular ações através de pessoas e não de protocolos de fluxos instituídos ${ }^{10,26}$.

Neste caso, é necessário atentar para as possíveis iniquidades em saúde que podem emergir pela falta de protocolos de fluxos instituídos em variados níveis de atenção à saúde, desta forma, ao discutir a articulação com a rede de saúde mental, que prima pelo cuidado em liberdade, a análise dos dados aponta para a materialidade da prática no cotidiano das ações. Por exemplo: como proceder com um usuário recém-chegado à cidade, andarilho, que apresenta um surto psicótico no Centro Pop? Quem é acionado? E quem responde de forma satisfatória a atenção e acolhimento psicossocial? CAPS? Hospital Geral? Qual fluxo estabelecido? É para a materialidade das ações no cotidiano das práticas, que este estudo aponta.

\section{Centro Pop e as cidades, por perfil populacional}

Ao considerarmos a maior concentração da PSR nas grandes cidades e centros urbanos ${ }^{7,14,15}$, apontamos a necessidade de pensar o acesso à saúde também como um direito a cidade, pois analisando os dados deste estudo foi demonstrada que a articulação entre os Centros Pop em relação aos CAPS e hospitais é ainda frágil principalmente nas metrópoles ${ }^{14}$. Grande parte das metrópoles e municípios de grande e médio porte também não possuem protocolo de fluxo para serviços de internação à PSR, o que já foi apontado como um possível problema na sessão anterior.

Em contrapartida, o estudo revelou que dentre os portes populacionais Metrópole, Grande e Médio, os municípios de grande porte tiveram mais respostas positivas principalmente em relação à variável protocolo de fluxo em sua articulação com os CAPS, sendo que a articulação com os hospitais se apresentou muito mais frágil. Este dado pode contribuir para a tomada de decisão dos agentes da esfera pública no que tange a maiores investimentos nas metrópoles e municípios de médio porte, havendo necessidade de aprofundamento sobre as causas da fragilidade na articulação com a rede de saúde mental destes municípios. Tal aprofundamento é relevante, pois é justamente nas metrópoles que se dá a maior concentração da PSR e paradoxalmente, é onde a rede se encontra mais frágil.

Ao resgatarmos o conceito de intersetorialidade como peça-chave deste debate, torna-se importante conhecer e reconhecer as dificuldades involucradas para garantir o direito à saúde para as populações mais vulneráveis, justamente onde e quando mais necessitem, no caso da PSR, nos espaços urbanos entendendo que "o direito à saúde se entrecruza com o direito à cidade na medida em que, ao se garantir espaços urbanos saudáveis para a população, também serão reduzidas iniquidades e expandido o usufruto dos efeitos positivos da urbanização a grupos postergados e desfavorecidos"16 (p. 3822).

\section{Conclusões}

Apesar dos claros avanços intersetoriais construídos no marco das políticas públicas para a população em situação de rua, este estudo aponta para uma rede intersetorial ainda frágil, como já sugerido por outros estudos, com uma moderada vantagem na articulação dos Centro Pop com os CAPS em detrimento da articulação com os serviços de urgência e emergência, principalmente nos municípios de grande porte, carecendo de melhora na articulação nas metrópoles e municípios de médio porte.

Apontamos a variável "Possui fluxo" como um importante analisador da função intersetorialidade exercida pelos Centro Pop, recalcando a importância de novos estudos quantitativos como complementos aos estudos qualitativos sobre o tema a fim de agregar dados novos. A escolha na realização de um estudo quantitativo se 
empenha em suprir a lacuna neste tipo de estudo, apontada pela literatura sobre o tema e em dar materialidade e visibilidade às problemáticas que envolvem as articulações no cuidado em saúde mental para a PSR no cotidiano das práticas. As dificuldades na prestação de cuidados em saúde à PSC são relatadas em diferentes estudos e continuam latentes na atualidade, o que torna relevante e excepcional, pensar na realização estudos longitudinais sobre a questão da intersetorialidade em saúde/saúde mental incluindo a variável "Fluxo".
Portanto as contribuições do presente estudo são também suas limitações, ou seja, revelam a importância dos protocolos de fluxo na atenção à PSR, mas não revelam as causas associadas às dificuldades nesta articulação. Faz-se necessária a continuidade das investigações sobre o tema e o investimento na formação de profissionais sensíveis à demanda complexa que é lidar com o sofrimento sociopolítico em talvez o que se apresente como sua máxima expressão (a falta de teto), o sofrimento psíquico e o cuidado em liberdade como premissa máxima do direito à cidade.

\section{Colaboradores}

TB Pedrosa foi responsável pela concepção do estudo, introdução, argumentação e justificativa, discussão e conclusões. P Cáceres-Serrano foi responsável pela definição e aplicabilidade do método de pesquisa e contribuiu para a análise dos dados. Ambos os autores são responsáveis pela redação e revisão final do artigo. 


\section{Agradecimentos}

À Pontifícia Universidad Católica de Valparaíso (PUCV), na figura do Professor Dr. Vicente Sisto, que através do Programa de Doutorado em Psicologia e da "Vicerrectoria de Investigación en Estudios Avanzados" (VRIEA), me permitiu obter a "Beca de Manutención para alumnos extranjeros de Programas de Doctorados", bolsa de estudos que permitiu ser possível minha estadia no Chile, mesmo pós revolta social e incidência da pandemia de COVID-19. Agradeço também às pessoas em situação de rua, sobretudo aos loucos e loucas de rua, que seguem inspirando a aposta na pesquisa acadêmica e no cotidiano das práticas.

\section{Referências}

1. Brasil. Ministério da Saúde (MS). Secretaria de Atenção à Saúde. Departamento de Atenção Básica. Coordenação-Geral de Planejamento e Vigilância Socioassistencial, editor. Manual sobre o cuidado à saúde junto à população em situação de rua. Brasil. Brasília: MS; 2012. p. 1-100.

2. Grandón FP, Vielma-Aguilera A, Castro-Alzate ES, Bustos NC, Saldivia BS. Caracterización de las personas en situación de calle con problemas de salud mental, que se encuentran en la Región del BíoBío. Rev Chil Neuropsiquiatr 2018; 56(2):89-99.

3. Wijk LB van, Mângia EF. Atenção psicossocial e o cuidado em saúde à população em situação de rua: uma revisão integrativa. Cien Saude Colet 2019; 24(9): 3357-3368.

4. Brasil. Lei n ${ }^{\circ} 10.216$ DE 2001 - Proteção direitos da pessoa portadora de transtornos mentais. Diário Oficial da União [Internet]. 2001; [acessado 2020 out 15]. Disponível em: http://hpm.org.br/wp-content/uploads/2014/09/lei-no-10.216-de-6-de-abril-de-2001. pdf

5. Brasil. Lei 8080/90. Dispõe sobre as condições para a promoção, proteção e recuperação da saúde, a organização e o funcionamento dos serviços correspondentes e dá outras providências. Diário Oficial da União [Internet]. 1990;1-13. [acessado 2020 out 15]. Disponível em: http://scholar.google.com/scholar?hl=en\&btnG=Search\&q=intitle:LEI $+\mathrm{N}^{\circ}+8.080,+-$ $\mathrm{DE}+19+\mathrm{DE}+\mathrm{SETEMBRO}+\mathrm{DE}+1990 \# 1$

6. Brasil. Política Nacional para Inclusão Social da População em Situação de Rua. [Internet]. 2008;25. [acessado 2020 out 15]. Disponível em: http://projetorua gempo.com.br/wp-content/uploads/2013/06/Pol?tica-nacional-para-inclus?o-social-da-popula??o-emsitua??o-de-rua.pdf

7. Brasil. Orientaçôes Técnicas: Centro de Referência Especializado para População em Situação de Rua - Centro Pop. 2011; 3:1-116.

8. Paiva IKS, Lira CDG, Justino JMR, Miranda MGDO, Saraiva AKDM. Direito à saúde da população em situação de rua: reflexões sobre a problemática. Cien Saude Colet 2016; 21(8):2595-2606.

9. Carneiro Junior N, Nogueira EA, Lanferini GM, Ali DA, Martinelli M. Serviços de saúde e população de rua: contribuição para um debate. Saude Soc 1998; 7(2):47-62. [acessado 2020 out 15]. Disponível em: http://www.scielo.br/scielo.php?script=sci_arttext\&pid=S0104-12901998000200005\&lng=pt\&tlng=pt

10. Borysow IC, Furtado JP. Acesso, equidade e coesão social: avaliação de estratégias intersetoriais para a população em situação de rua. Rev da Esc Enferm da USP 2014; 48(6):1069-1076.

11. Carmo ME, Guizardi FL. Desafios da intersetorialidade nas políticas públicas de saúde e assistência social: uma revisão do estado da arte. Physis Rev Saude Coletiva 2017; 27(4):1265-1286.

12. Akerman M, Sá RF, Moyses S, Rezende R, Rocha D. Intersetorialidade? Intersetorialidades! Cien Saude Colet 2014;19(11):4291-4300.

13. Junqueira LAP. Intersetorialidade, transetorialidade e redes sociais na saúde. Rev Adm Publica 2000; 34(6):35-45. 
14. Varanda W, Adorno RCF. Descartáveis urbanos: discutindo a complexidade da população de rua e o desafio para políticas de saúde. Saude Soc 2004;13(1):56-69.

15. Vale AR, Dalla Vecchia, M. "UPA é nós aqui mesmo": as redes de apoio social no cuidado à saúde da população em situação de rua em um município de pequeno porte. Saude Soc 2019; 28(1):222-234.

16. Figueiredo GLA, Martins CHG, Damasceno JL, Castro GG de, Mainegra AB, Akerman M. Direito à cidade, direito à saúde: quais interconexões? Cien Saude Colet 2017; 22(12):3821-3830.

17. Brasil. Coordenação-Geral de Planejamento e Vigilância Socioassistencial. Validez CENSO SUAS 2019. 2020.

18. Brasil. Censo SUAS 2019. 2019.

19. Greennacre M. La práctica del análisis de correspondencias. Fundación BBVA, editor. Madrid; 2008.

20. Husson F, Lê S, Pagès J. Exploratory Multivariate Analysis by Example Using. $2^{\mathrm{a}}$ ed. Chapman Hall/CRC Comput Sci Data Anal.; 2017.

21. Vivanco M. Análisis estadístico multivariable. Editorial. Santiago de Chile; 1999.

22. Lê S, Josse J, Husson F. FactoMineR: An R Package for Multivariate Analysis. J Stat Softw 2008; 25(1):1-18.

23. Rosa AS, Secco MG, Brêtas ACP. O cuidado em situação de rua: revendo o significado do processo saúdedoença. Rev Bras Enferm 2006; 59(3):331-336.

24. Pedrosa TB, Moreira MIB. Saúde mental e justiça: os desafos do cuidado em rede. Rev Bras Pesqui em Saúde 2018; 19(2):73-82.

25. Sicari AA, Zanella AV. Pessoas em Situação de Rua no Brasil: Revisão Sistemática. Psicol Cienc Profissão 2018; 38(4):662-679.

26. Serafino I, Luz LCX. Políticas para a população adulta em situação de rua: questões para debate. Rev Katálysis $2015 ; 18(1): 74-85$.

Artigo apresentado em 31/10/2020

Aprovado em 15/10/2021

Versão final apresentada em 17/10/2021

Editores-chefes: Romeu Gomes, Antônio Augusto Moura da Silva 